\title{
An Approach to Plasma Wake Studying
}

\author{
Gennadii V. Gembarzhevskii \\ Russian Academy of Sciences, The Institute for Problems in Mechanics, Moscow, Russia \\ Email: gvgemb@ipmnet.ru
}

Received 30 October 2014; accepted 11 November 2014; published 22 November 2014

Copyright (C) 2015 by author and Scientific Research Publishing Inc.

This work is licensed under the Creative Commons Attribution International License (CC BY).

http://creativecommons.org/licenses/by/4.0/

(c) (i) Open Access

\section{Abstract}

The influence of a glow electrical discharge on the wake behind two cylinders was studied. This effect consists in a redistribution of the power in the velocity pulsation spectrum due to the discharge action on fluid flow. To treat this observation, we need a simple model of intermittent turbulent wake in the vicinity of cylinders. A variant of such model has been developed in the form of two coupled van-der-Pole oscillators representing two interacting von Karman vortex streets behind the cylinders. According to the model, the set of the global wake modes and its concurrence are discussed. Accordingly, a mechanism of the glow discharge effect on the cylinders wake has been proposed.

\section{Keywords}

Turbulentplasma Wake, Alternation, Over-Heating Instability, Reduced-Order Model, Van-der-Pole Oscillators, Vortex Streets Synchronization

\section{Introduction}

During last century the wake behind cylinders attracted considerable attention and now the research for that is in progress. Such contemporary investigations are of various types, namely, of experimental, numerical, theoretical, and complex ones [1]-[9]. Some recent topic is a flow control over moving body for various aims, such as a delay of laminar regime, or triggering flow separation, heat- and mass-flux modification, etc. Among various types of appropriate actuator for flow control an electrical ones have sufficient advantages, such as the fast response, reliability, low cost [10] [11]. Accordingly, dielectric barrier and corona discharges were intensively studied for such purposes. Among many of them, few examples of flow modification by means of plasma actuators around bluff (cylinders) and smooth bodies (wings) were described in references [12]-[15].

The problem of our direct interest is in the field of plasma wakes, more exactly, turbulent flows in electrical constant power industrial lasers of $\mathrm{kW}$ class, some relevant references to this topic are in papers [16]-[19]. We tried to adopt the technique of flow control by the electrical discharge in the fast-flow $\mathrm{CO}_{2}$-laser where the glow 
discharge exists ab initio. A promising result has been achieved for this case [20]. In that study the wake from two parallel circular cylinders in the side-by-side arrangement was studied for the mixture composed of 16 Torr nitrogen and 2 Torr helium, see Figure 1. The cross section of the discharge chamber was of $55 \times 940 \mathrm{~mm}$, the diameter of the quartz cylinders was of $15 \mathrm{~mm}$ while the diameter of the copper water-cooled cathode was of 4 $\mathrm{mm}$. The cylinders touched the cathode. This cylinders wake may be treated as a incompressible turbulent flow since the value of the flow Mach number was of $M<0.15$ and the value of Reynolds number defined in terms of cylinder diameter $D$ and non-perturb velocity was Re $\sim 1000$. Mean velocity was measured by means of Prandtl tube. One-point spectra of the wake velocity were measured in $120 \mathrm{~mm}$ beneath the axis of one cylinder by means of a transducer of Siddon-Ribner type [21]. Its signal was processed by a PALSE 3560C complex of Bruel \& Kjar. Experimental velocity spectra for the wake at non-self-sustained glow discharge and appropriate spectra for the neutral gas flow (that is a reference data) were compared. When the distance between cylinder axes was about $L / D \approx 2.2$ as is in Figure 2, the velocity spectrum with one peak was observed in the case of gas wake. This result was treated as a manifestation of the presence of one global hydrodynamic mode existing in the case of gas wake with above mentioned parameters. On the contrary, for a relevant plasma wake flow the two-peak velocity spectrum was observed and interpreted as a property of intermittent flow of two concurrent global modes. One of global modes of the plasma wake was the same as for neutral gas flow (since its oscillation frequency was the same), whereas another new mode had lower frequency as compared to previous one. When current density at cathode tube was of about $2 \mathrm{~A} / \mathrm{m}$ (the case in Figure 2(b)) the dominant part of the energy of coherent oscillations was concentrated in the lower frequency peak. Consequently, this discharge influence appeared to be strong.

\section{Model of the Wake behind Two Cylinders}

For treatment of the discharge effect one has to use some model of the wake. Moreover, when the flow visualization is difficult as it is in our case, even spectral peak classification (of one-point velocity record) requires for some model of the wake. Accordingly our first purpose was developing wake model, no matter appreciable for neutral gas wakes only, but with wide applicability area in Reynolds number variation and in the cylinder spacing alternation. Desirable model has to be the most simple yet leaves room for further extension to such factors as electric discharge influence. Simple wake models are reduced-order models of Landau type, for example [22]. This model is well-studied and justified for low value of supercriticality $\left(\operatorname{Re}-\operatorname{Re}_{c r}\right) / \operatorname{Re}_{c r} \leq 1, \operatorname{Re}_{c r} \sim 50-100$. In Russia Prof. P. Landa develops similar reduced-order oscillatory models for various physical systems including wakes (with Prof. A. Ginevsky) as well [23]. We used such approach for turbulent wake modeling with van-derPole equation as the starting point, accordingly, no immediate limitation on Reynolds number arises for this option. In experiment [20] in the chamber of fast-flow $\mathrm{CO}_{2}$-laser the cylinders of low aspect ratio were used: $H / D \sim 3.5$. This circumstance allows us neglect the wake dynamics in direction of cylinders axes, according to results [24]. On the author hand, it is desirable to have model appreciable for wide range of interaction intensity of von Karman streets. Then the developed model of the wake from two cylinders with side-by-side arrangement is a set of two dimensionless differential equations for coupled van-der-Pole oscillators representing two interfering Benard-von Karman streets behind cylinders in proximity (how the streets were worked out by the end of the formation zone):

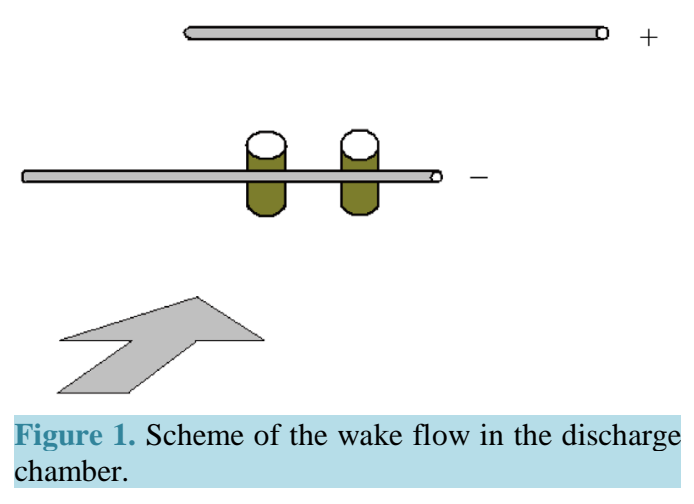




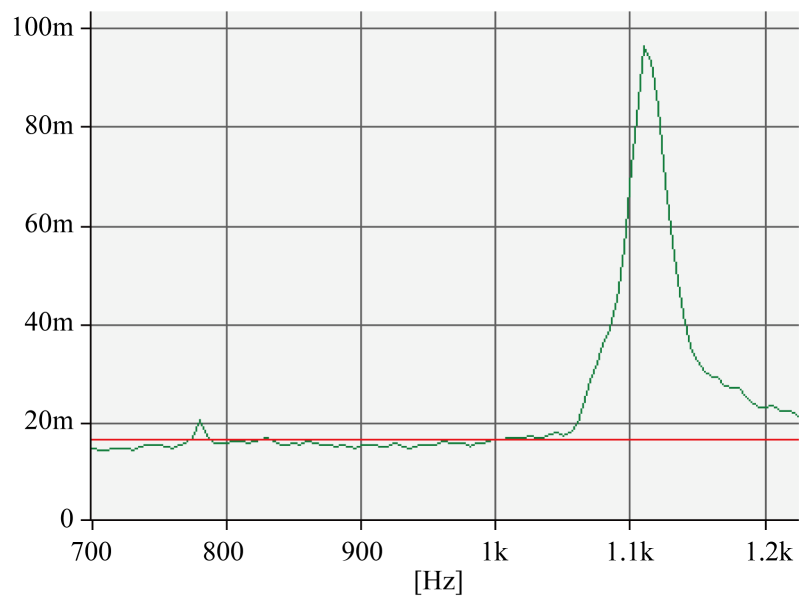

(a)

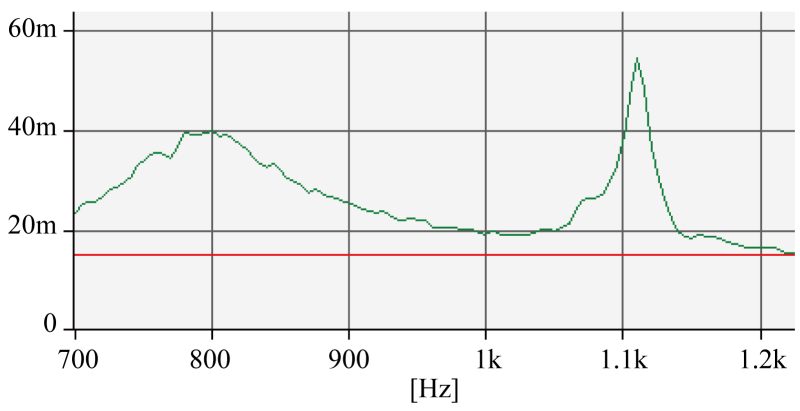

(b)

Figure 2. Spectra of the wake velocity pulsations (arbitrary units): for neutral gas flow (a), and for discharge plasma wake (b).

$$
\begin{aligned}
& \frac{\mathrm{d}^{2} X_{1}}{\mathrm{~d} t^{2}}+X_{1}-\varepsilon\left[1-X_{1}^{2}-\lambda X_{2}^{2}-l X_{1} X_{2}\right] \frac{\mathrm{d} X_{1}}{\mathrm{~d} t}=h_{1} \\
& \frac{\mathrm{d}^{2} X_{2}}{\mathrm{~d} t^{2}}+X_{2}-\varepsilon\left[1-X_{2}^{2}-\lambda X_{1}^{2}-l X_{1} X_{2}\right] \frac{\mathrm{d} X_{2}}{\partial t}=h_{2}
\end{aligned}
$$

Here the oscillator coordinates $X_{1}$ and $X_{2}$ represent the transversal components of the flow velocity in two von Karman streets behind two cylinders respectively. Right-hand sides of the equations describe random forces executed on the zone of street formation by the turbulence of incoming flow. Equation set $(1,2)$ has three dimensionless parameters. Precisely, $\varepsilon$ is the native nonlinearity parameter of the partial van-der-Pole oscillator-street, $\lambda$ and $l$ are two parameters of the oscillators cross damping. Here, the peculiarity of this model should be underlined. In an attempt to extend the applicability area of the model, the oscillators coupling form was made nonlinear, accordingly, the term in brackets in set $(1,2)$ is a square polynomial of general form. Assuming quasi-stationary state of the wake behind the formation zone, the model transforms to a one-dimensional type through the transformation $t \rightarrow t-x_{\text {wake }} / u_{\text {translation }}$ as it is done for Landau model [22]. Moreover, direct experimental evaluation of nonlinearity parameter $\varepsilon$, by treating velocity oscillograms of transient wake regimes, led to value $\varepsilon=2 \varepsilon_{[25]} \sim 0.3$ for our case [20] [25] and new notation of set (1,2). As parameter $\varepsilon$ is small the Poincare-Krylov-Bogolyubov method of slow amplitudes and phases [26] has been applied for solving the set (1, 2) by using the form:

$$
\begin{aligned}
& X_{1}=\rho \cos (\theta+t) \\
& X_{2}=r \cos (\psi+t)
\end{aligned}
$$

Here $\rho$ and $r$ are the amplitudes whereas $\theta$ and $\psi$ are the phases of the first and the second oscillator, respectively. Then, corresponding set of "truncated" equations (that means a neglect of the small impact of the 
overtones in the case $\varepsilon \rightarrow 0$ ) can be written as

$$
\begin{aligned}
& \frac{\partial \rho}{\partial t}-\frac{\varepsilon \rho}{2}\left[1-\frac{\rho^{2}+\lambda(2-\cos (2 \varphi)) r^{2}+l \rho r \cos (\varphi)}{4}\right]=h_{\rho} \\
& \frac{\partial r}{\partial t}-\frac{\varepsilon r}{2}\left[1-\frac{r^{2}+\lambda(2-\cos (2 \varphi)) \rho^{2}+l \rho r \cos (\varphi)}{4}\right]=h_{r} \\
& \frac{\partial \varphi}{\partial t}+\frac{\varepsilon}{8}\left[\lambda\left(\rho^{2}+r^{2}\right) \sin (2 \varphi)+2 l \rho r \sin (\varphi)\right]=h_{\varphi} .
\end{aligned}
$$

Here, it was introduced the phase difference $\varphi$ for two oscillators-streets

$$
\varphi=\theta-\psi
$$

Equations set (5-7) for the case of zero right-hand sides $h_{i}=0$ may be analyzed by seeking for the "laminar" global wake modes, and this work was originally performed by us. The results of this analysis were presented at XII Moscow Workshop on Magneto-Plasma Aerodynamics-2013 [27]. But at this point it is useful to make a change of dependent variables for simplifying the model set. This transformation is

$$
\begin{aligned}
& D=(1 / 2)\left[1-\frac{2 \rho r}{\left(\rho^{2}+r^{2}\right)}\right] \\
& K=\cos (\varphi) \\
& R^{2}=\rho^{2}+r^{2}
\end{aligned}
$$

Accordingly, the model of the wake behind the cylinders pair in the side-by-side arrangement takes the final form:

$$
\begin{aligned}
& \frac{\mathrm{d} K}{\mathrm{~d} t}-\frac{\varepsilon R^{2}}{4}\left[1-K^{2}\right]\left[\lambda K+\frac{l}{2}(1-2 D)\right]=h_{K} \\
& \frac{\mathrm{d} D}{\mathrm{~d} t}-\frac{\varepsilon R^{2}}{4}\left[\lambda\left(3-2 K^{2}\right)-1\right]\left[D-3 D^{2}+2 D^{3}\right]=h_{D} \\
& \frac{\mathrm{d} R^{2}}{\mathrm{~d} t}-\varepsilon R^{2}\left[1-R^{2} \frac{2+l K(1-2 D)+\left[\lambda\left(3-2 K^{2}\right)-1\right](1-2 D)^{2}}{8}\right]=h_{R}
\end{aligned}
$$

One can see that the developed model (Equations (12-14)) turns out to be relatively simple. Actually, small nonlinearity parameter $\varepsilon$ may be excluded through the transformation $t \rightarrow \varepsilon t$ then the model becomes a twoparametric one with parameters $\lambda$ and $l$. Moreover, the first two equations for $K$ and $D$ evolution are practically independent of the third equation for $R$ dynamics. According to the sense of the model, stable stationary points of Equations (12-14) with zero right-hand sides $h_{i}=0$ represent global modes of the wake. Then the system of the wake modes are i) - iv).

i) Asymmetric mode " AS " with one von Karman street of the complex wake is damped. It has the form:

$$
\rho=2 ; r=0 \text { or } r=2 ; \rho=0 .
$$

The domain of existence of AS mode is

$$
\lambda>1
$$

ii) The second one is symmetric mode " $S_{0}$ ” of in-phase synchronized equal oscillators-streets. Its form is:

$$
\rho=r=2 / \sqrt{1+\lambda+l} ; \quad \varphi=0
$$

The domain of existence of $S_{0}$ mode is: 


$$
\lambda<1 ; \quad l>-2 \lambda
$$

iii) Symmetric mode " $S_{\pi}$ " of anti-phase synchronized von Karman streets has the form:

$$
\rho=r=2 / \sqrt{1+\lambda-l} ; \quad \varphi=\pi
$$

The existence domain of this mode is as follows:

$$
\lambda<1 ; \quad l<2 \lambda
$$

iv) Symmetric mode " $S_{\varphi}$ ” consists of two equal von Karman streets synchronized by angular $\varphi$ depended on $\lambda$ and $l$ parameters. This mode has the form:

$$
\rho=r=\frac{2}{\sqrt{1+3 \lambda-l^{2} / \lambda}} ; \quad \varphi=(+/-) \arccos \left(-\frac{l}{2 \lambda}\right)
$$

The global mode $S_{\varphi}$ exists in a simply connected domain

$$
-(1 / 3)<\lambda<0 ; \quad|l|<-2 \lambda \text { and } \lambda<-(1 / 3) ; \sqrt{3 \lambda^{2}+\lambda}<|l|<-2 \lambda
$$

It should be mentioned that the modes $A S$ and $S_{\varphi}$ are twice degenerate ones according to Equations (15) and (21). For convenience the existence map on the $\lambda l$-plane for the wake modes is presented in Figure 3.

Remark (1): In the area VI in Figure 3: $\lambda<-1 ;|l|<\sqrt{3 \lambda^{2}+\lambda}$ an infinitely growing mode arises. Remark (2): Three modes $S_{0}, S_{\pi}$ and $S_{\varphi}$ demonstrate the effect of oscillation synchronization of two parts of complex systems in the case of weak interaction force between them, see [23] [28]. The asymmetric mode AS belongs to an opposite case of strongly coupled subsystems. In summary, the two-parametric $\lambda l$-model developed is rich enough to cover four global modes of the cylinder parewake, two of them are twice degenerate.

\section{Modeling the Intermittent Turbulent Wake}

\subsection{Intermittent Wake of Weakly Coupled Streets}

From the map in Figure 3 one can easily see that the alternating wake may be observed in wide triangle III:

$$
0<\lambda<1 ; \quad|l|<2 \lambda
$$

Moreover, these are modes of in-phase $S_{0}$ and anti-phase $S_{\pi}$ synchronized streets that may coexist in this triangle of parameters. Next step of the model development is a calculation of the alternation coefficient for these two wake modes. With this aim one has to trace the attraction domains of $S_{0}$ and $S_{\pi}$ modes in the model

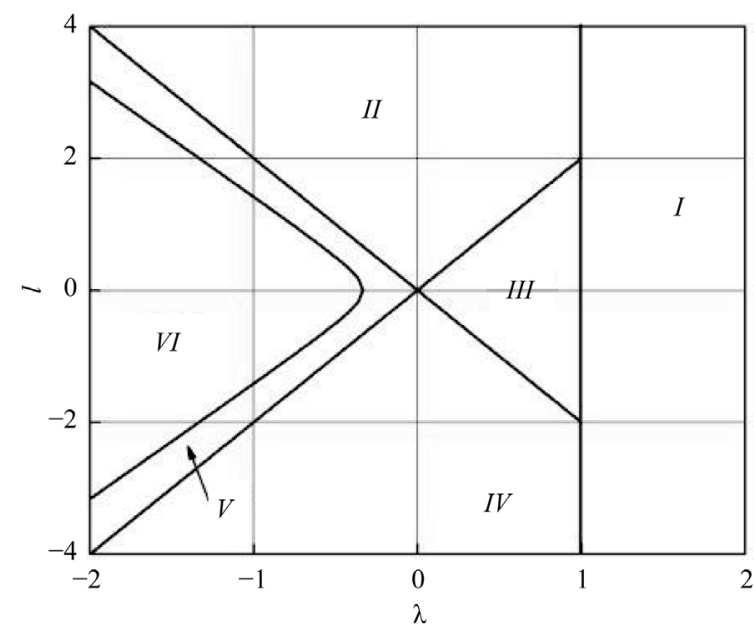

Figure 3. Map of regimes of two-cylinder wake: the mode AS exists in the domain I; $S_{0}$ in II; $S_{0}$ and $S_{\pi}$ in III; $S_{\pi}$ in IV; $S_{\varphi}$ in $\mathrm{V}$; VI is a nonphysical domain. 
phase space. This task may be achieved by taking into consideration the following arguments. The boundary of these domains on $K D$ plane is a certain trajectory of the model set (12-14). Due to this fact, the equation for the boundary takes the form

$$
\frac{\mathrm{d} K}{\mathrm{~d} D}=\frac{\left[1-K^{2}\right][(l / 2)(2 D-1)-K \lambda]}{\left[1-\lambda\left(3-2 K^{2}\right)\right]\left[D-3 D^{2}+2 D^{3}\right]}
$$

This special trajectory must joint unstable stationary points of set $(12-14)$ with $h_{i}=0$, then the necessary boundary condition for Equation (24) may be written as

$$
K_{(0)}=-(l / 2 \lambda) \text {, and } K_{(1 / 2)}=0
$$

In real time the motion along the boundary is unstable. Accordingly, one has to distinguish between three possibilities when numerical calculating this boundary. In the first case, for the domain of the model parameters $0<\lambda<(1 / 3) ;|l|<2 \lambda$, the boundary condition to Equation (24) must be given at the first point (25) in the form $K_{(0)}=-(l / 2 \lambda) ; \frac{\partial K}{\partial D}{ }_{(0)}=\frac{l\left[1-\left(l^{2} / 4 \lambda^{2}\right)\right]}{\left[1-2 \lambda+\left(l^{2} / 4 \lambda\right)\right]}$, as it is a saddle point. For the second aria of parameters $\frac{1}{3}<\lambda<1 ;\left|\frac{l}{2 \lambda}\right|<\sqrt{\frac{3-(1 / \lambda)}{2}}$, the boundary condition has to be given at the second point (25) in the form $K_{(1 / 2)}=0 ;{\frac{\partial K}{\partial D_{(1 / 2)}}}=\frac{2 l}{(5 l-1)}$ (as well a saddle point for the case). And in the third case $\frac{1}{3}<\lambda<1 ; \sqrt{\frac{3-(1 / \lambda)}{2}}<\left|\frac{l}{2 \lambda}\right|<1$ one must account for two solutions from both boundary points (25). Those solutions merge at the point $K_{k}=-\frac{l}{|l|} \sqrt{\frac{3-(1 / \lambda)}{2}}, D_{k}=\frac{1}{2}\left[1-\frac{\sqrt{2 \lambda(3 \lambda-1)}}{|l|}\right]$ which is an unstable knot in the case. Some examples of numerical calculation of boundary curve are presented in Figure 4.

Here some comment may be done. The boundary curve $K_{(D)}$ appears to be a monotonically increasing function of its variable $D$ for positive values of its parameter $l:|l|<2 \lambda$ and is a mirror-symmetry decreasing function of $D$ for negative values of parameter $l$ since $K$ is an odd function of parameter $l$ according to the model set (12), (13), (14). It seems physically natural that the appearing probabilities for modes $S_{0}$ and $S_{\pi}$ occurs to be equal for the case $D \rightarrow 1 / 2$, i.e. the boundary curve in Figure 4 approaches the value $K \approx 0$ when the initial representation point in the phase space is far from both attraction points $(D=0 ; K=+/-1)$. But these probabilities diverge from value $50 \%$ if the initial representation point moves closer to the attraction points ( $D \rightarrow 0$ in Figure 4). Naturally, the sign of this separation depends on the sign of the parameter $l$ that may be easily seen from the form of the last bracket in the phase dynamic Equation (12).

Now we are ready to evaluate the alternation coefficient of the wake within the framework of the model. Let the turbulence intensity of incoming flow be large. This factor is modeled by the right-hand sides of the Equations (1), (2), Equations (5)-(7) and Equations (12)-(14) according to the model developed. Then, for the case of strong interruptions regime, the mean residence time for each compete mode would be proportional to the area of the attraction domains for corresponding mode. Hence, for this case of strong incoming turbulence, the alternation coefficient of the wake may be defined as:

$$
\Gamma_{\pi}=\frac{1}{2}+\int_{0}^{0.5} K \mathrm{~d} D
$$

Here this alternation coefficient is defined by the emergence of the mode of anti-phase synchronized streets. (Somebody may prefer the coefficient $\Gamma_{0}=1-\Gamma_{\pi}$ defined by the emergence of the mode of in-phase synchronized streets.)According to definition (26) and the problem formulation $(24,25)$, the alternation coefficient $\Gamma_{\pi}$ may be calculated numerically. Another opportunity reduces to developing an analytical approximate solution of the boundary problem $(24,25)$ in the form of a truncated expansion. Now the main interest is in the wake of 


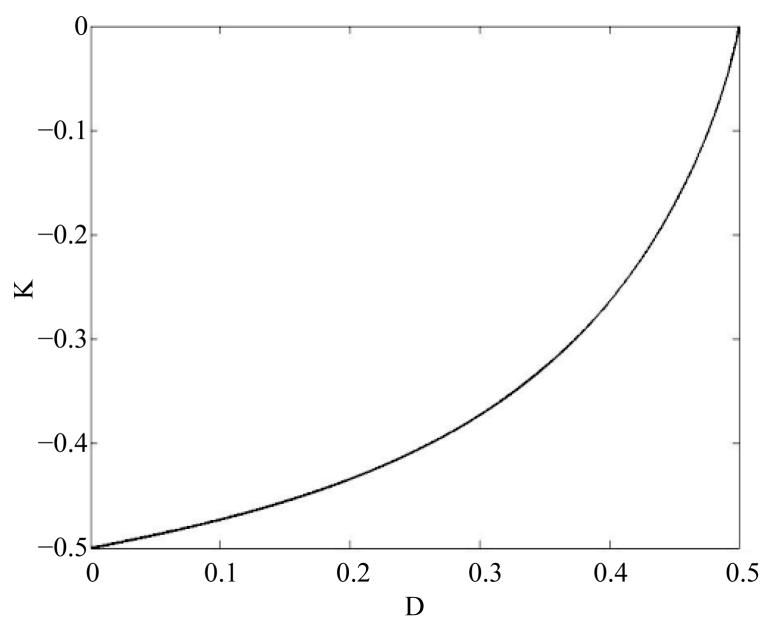

(a)

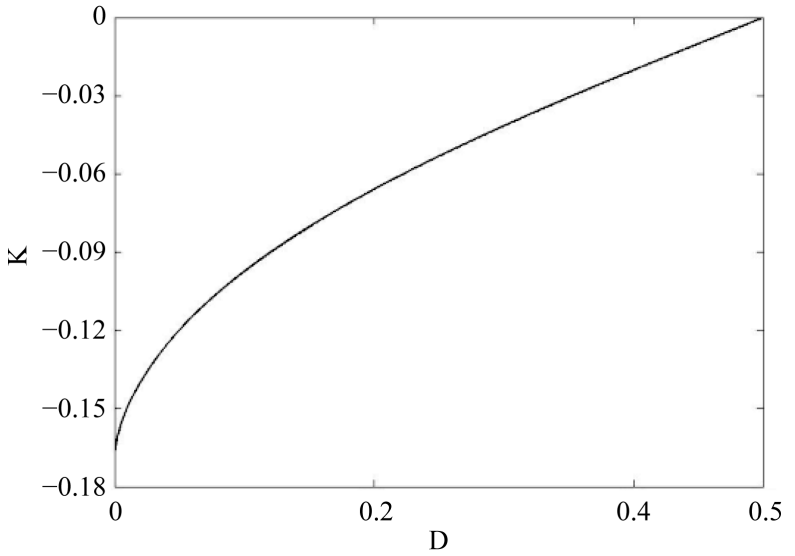

(b)

Figure 4. Boundary of the attraction domains of modes in the phase space: (a) for case $\lambda=0.2 ; l=0.2$, (b) for case $\lambda=0.6 ; l=$ 0.2 .

weakly coupled von Karman streets. In the case of first domain of parameters $0<\lambda<(1 / 3)$; $|l|<2 \lambda$ one may use an expansion in small parameter $\lambda$.

$$
K=K_{0}+K_{1} \lambda+K_{2} \lambda^{2}+\cdots
$$

By this way one comes to relevant expansion of the alternation coefficient $\Gamma_{\pi}$ with the same small parameter.

$$
\begin{gathered}
\Gamma_{\pi}=\frac{1}{2}\left[1+\alpha_{(p, \lambda)} p\right] \\
\alpha \approx-1+\lambda(2 \ln 2)\left(1-p^{2}\right)+\lambda^{2} 4\left(I+J p^{2}\right)\left(1-p^{2}\right)+\cdots
\end{gathered}
$$

Here the new parameter $p$ and constants $I$ and $J$ are introduced. Their definitions are following

$$
\begin{gathered}
p=\frac{l}{2 \lambda} \\
I=\int_{0}^{1 / 2} \mathrm{~d} y \int_{0}^{y} \frac{3 x-\ln (1-x)+\ln (1-2 x)}{x(1-x)(1-2 x)} \mathrm{d} x \approx 0.217 \\
J=\int_{0}^{1 / 2} \mathrm{~d} y \int_{0}^{y} \frac{(4 x+1)[\ln (1-x)-\ln (1-2 x)]-2 x}{x(1-x)(1-2 x)} \mathrm{d} x \approx 1.294
\end{gathered}
$$


Results of such numerical (dots) and analytical (solid curves) evaluation of the alternation coefficient $\Gamma_{\pi}$ are presented in Figure 5.

Remark (1): Alternation coefficient is an odd function of parameter $l$ or $p$ due to the symmetry properties of the model, accordingly, the data adduced in Figure 5 are for the case of positive interval of $p, l$ variation only. Remark (2): It should be noted that the difference between analytical data $(28,29)$ and numerical results is not so large even for maximum value of the expansion parameter $(\lambda=0.333)$. Summary of the results is as follows. The alternation coefficient $\Gamma_{\pi}$ of $S_{0}$ and $S_{\pi}$ wake modes in domain $0<\lambda<(1 / 3) ;|l|<2 \lambda$ varies in the interval $(-1 ; 1)$. It relatively strongly depends on the first model parameter $p$, whereas its dependence on the second parameter $\lambda$ is relatively weak. Precisely, $\Gamma_{\pi}$ is a monotonically decreasing function of $p$. This feature follows from the first boundary condition (25) for the equation (24): $K_{(0)}=-p$. And the alternation coefficient $\Gamma_{\pi}$ is a monotonically increasing function of $\lambda$ for the case $p>0$.

\subsection{Intermittent Wake of Strongly Coupled Streets}

When considering the case of strongly coupled von Karman streets $\lambda \geq 1$, one can recognize some other possibility for intermittent regime existence within the framework of the model. From the map in Figure 3 it can be seen that this intermittent regime may realize at the line

$$
\lambda=1
$$

Fortunately, for this case, the general solution for the phase trajectory on $K D$ plane may be written in explicit form

$$
K=\frac{l}{1-2 D}[C \sqrt{D(1-D)}-1 / 2]
$$

Here $C$ is an arbitrary constant. Figure 6 is the illustration of phase trajectories for the case. Here points $K=1 ; D=1 / 2$ and $K=-1 ; D=1 / 2$ are stable, accordingly mode $A S$ is also stable. Moreover, there are quasi-stable states within the framework of the model for the case $\lambda=1$. These quasi-stable states are not points but intervals

$$
K=1 ; D \in(0 ; 1 / 2) \text { and } K=-1 ; D \in(0 ; 1 / 2), \text { for the case }|l|<2
$$

In the case $l>2$ these states exists in two intervals

$$
D \in(0 ; 1 / 2), K=1 ; \text { and } D \in(1 / 2-1 / l ; 1 / 2), K=-1
$$

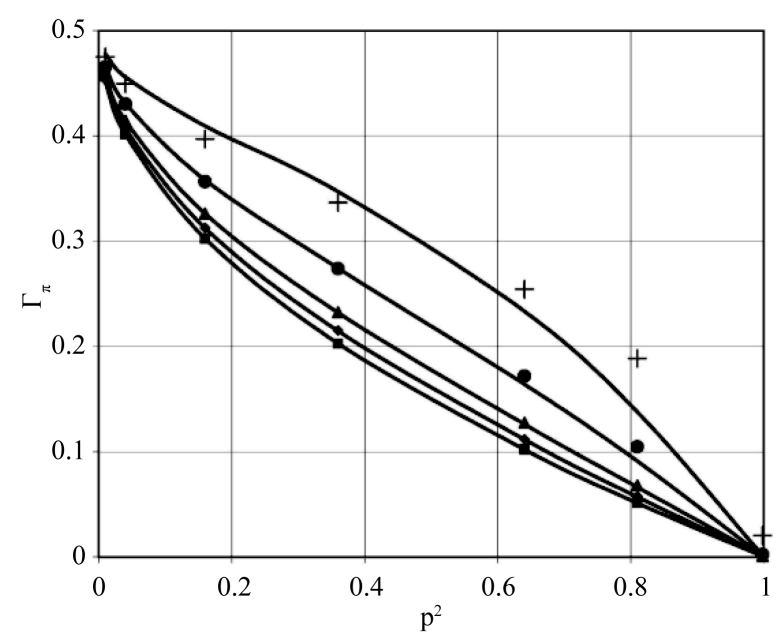

Figure 5. Intermittence coefficient $\Gamma_{\pi}$ as a function of the model parameters $p=1 / 2 \lambda$ and $\lambda$. The last parameter monotonically grows from low curve to top curve: $\lambda=0.01 ; 0.05 ; 0.1 ; 0.2$; 0.333 , respectively; whereas the first parameter $p$ is positive for all curves. 


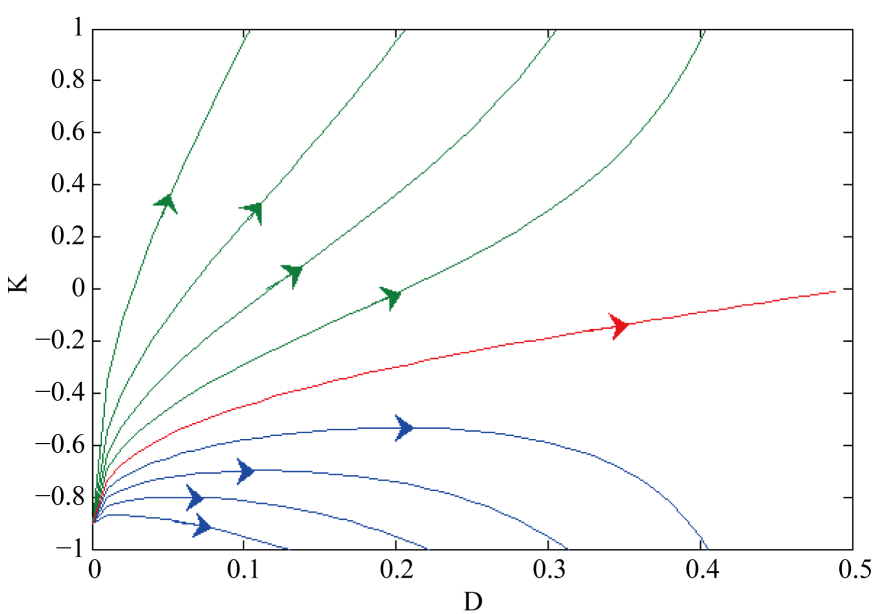

(a)

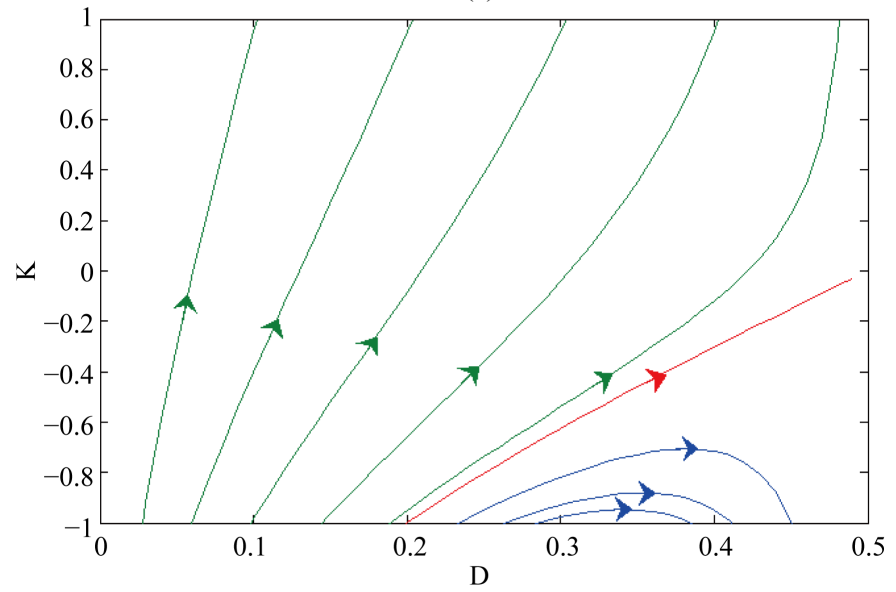

(b)

Figure 6. Oscillators-streets dynamics for the case $\lambda=1$; (a) for case $l=$ 1.8 , (b) for case $l=6$.

For symmetric case $l<-2$ one has intervals of existence

$$
D \in(1 / 2+1 / l ; 1 / 2), K=1 ; \text { and } D \in(0 ; 1 / 2), K=-1
$$

These conclusions are easily seen from the form of general solution (34) of dynamic equations illustrated by Figure 6. Here upper line $(K=1)$ represents quasi-stable mode of in-phase synchronized streets of different strength $(D \neq 0)$, when lover line $(K=-1)$ corresponds to anti-phase synchronized quasi-stable streets of different strength. Let these modes of two unequal streets be named ${ }^{*} A S_{0}$ and $* A S_{\pi}$, respectively. For clearness, the phase trajectories ending at ${ }^{*} A S_{0}$ wake mode are market green when those ending at ${ }^{*} A S_{\pi}$ mode are market blue in Figure 6, and the separatrix is red. So, within the framework of the model, the only case when strongly coupled von Karman streets may alternate is the line $\lambda=1$. Three modes alternate at this one-dimensional domain, namely: stable mode $A S$ and two quasi-stable modes $* A S_{0}$ and $* A S_{\pi}$.

\section{Approbation of the Model}

The multiplicity of wake modes existing for two cylinders in side-by-side configuration is well known from the visualization experiments and as well as from simulations. The developed model managed to cover four global modes of the cylinders wake. There are modes of in-phase and anti-phase synchronized streets among four of them. It is known that these modes exist in the case of a relatively weak interaction of the von Karman streets formed by each cylinder when the cylinders are not so close to each other (see, for example [3] [29]). And the 
intermittent wake regime of these two modes is known for some wake parameters, as well [29]. Our two-dimensional wake model also brings to a restriction of existence domain of $S_{\pi}$ mode at low $L / D$ ratio but not at high $L / D$ values [30]. All this facts related to modes of in-phase and anti-phase synchronized streets appear to be in a good agreement with our one-dimensional model predictions. The third mode of the model AS is an asymmetric mode with one of the vortex streets damped. It must be attributed to the case of relatively strong interaction of von Karman streets when "deflected flow" and "flip-flop" regimes were visualized and evaluated, for example in references [3] [22] [31] [32]. The last fourth mode of the model $S_{\varphi}$ represents the wake in the form of two equal streets synchronized by the angle being not equal to 0 or $\pi$. Some similar wake configurations may be observed. Then the question of whether they are stable or transient arises? Existence of two quasi-stable modes $* A S_{0}$ and $* A S_{\pi}$ do not contradict to observations and simulations as well. The model developed is not unique one among other one-dimensional models for the wake from cylinders group. Commonly, some kind of Stuart-Landau model (Landau-Ginzburg model) is used for the wake from cylinders. The earlier papers [22] [32] [33] are examples. An advantage of our wake model as compared to the Stuart-Landau models is a low number of its parameters (only two) in combination with nonlinear form of the street interaction term with these parameters quantity. Therefore, we hope that the domain of its validity is wider as compared to the domain of Stuart-Landau models with linear term of the street coupling and restricted validity aria for the flow Reynolds number.

\section{Model Application to Plasma Wake Studying}

Formally, one has two possibilities when applying the model to cylinders intermittent wake. These are the cases of weekly (3.1) and strongly (3.2) coupled streets as it was discussed above. The variant of weekly interacting streets is not our case as the frequency splitting of the two observed modes was large enough $~ 30 \%$ (see Figure 2) for $S_{0}$ and $S_{\pi}$ alternation. Moreover, for such small spacing as $L / D \approx 2.2$ in our experiment [20], the mode of anti-phase synchronized streets $S_{\pi}$ was not observed as it may be concluded from the literature. Accordingly, it only remains to use the case of strongly coupled streets (3.2) with alternating modes AS and $S_{0}$ (or may be $S_{\pi}$ ) when describing intermittent plasma wake within the framework of the model for pitch ratio $L / D \approx 2.2$ in the case. Then, what is the role of the glow electrical discharge in the wake switching from $S_{0}$ global mode (or $S_{\pi}$ mode) to intermittent regime of $A S$ and $S_{0}$ (or $S_{\pi}$ ) modes? Nonhomogeneous wake heating due to energy release through the discharge may act as some equivalent of the lowering of the cylinders spacing. Consequently, glow discharge switching-on influences on the model parameters. Supposedly, the discharge switching-on increases the model parameter $\lambda$ similarly to the cylinders spacing influence. Consequently, if the value of the wake parameter $\lambda \approx 1$, one may switch over the wake regime by means of discharge power regulation. Here the interplay of the symmetry properties of the wake hydrodynamic modes and the discharge property of homogeneity breaking at greater currents must be accounted for. Suppose that the parameters of the neutral gas wake lie at the range $0<1-\lambda \ll 1$. Hence, the wake state is described by the stable mode $S_{0}$ (or $S_{\pi}$ ) according to the model whereas the mode $A S$ is unstable. The same situation is for plasma wake with sufficiently low currents. But for greater currents the development of the plasma overheating process [16] in the case of asymmetric hydrodynamic mode $A S$ changes the parameter $\lambda$ to such values as $0<\lambda_{A S}-1 \ll 1$. It means that the plasma wake at the $A S$ mode becomes stable for such elevated currents, yet the plasma wake at $S_{0}$ (or $S_{\pi}$ ) mode remains to be stable $0<1-\lambda_{S_{0}} \ll 1$ since the current distribution is more homogeneous due to more homogeneous hydrodynamic parameters distribution of the mode $S_{0}$ as compared to the distribution of the AS mode. Then the $A S$ - and $S_{0}$-modes begin to alternate due to the turbulence of incoming flow. Moreover, one may suppose that the model parameter $\lambda$ shift between two intermitting modes must be greater for the greater currents, since overheating is a nonlinear process. Thus, one observes a growth of the wake alternation coefficient $\Gamma_{A S}$ as the discharge current increases [20].

Realization ability of this scenario depends on amount of the plasma heating effect. The redistribution of power in the velocity spectra was observed at relatively low discharge power $W \sim 2000 \mathrm{~V} \times 2 \mathrm{~A}$ [20]. For an evaluation of discharge influence on the wake one may initially consider the case of the homogeneous distribution of the current density across the chamber and complete vibration relaxation of nitrogen (in collisions with molecular of uncontrolled admixture). Then this heating would be $\Delta T \sim 60 \mathrm{~K}$ to the end of the chamber for our case of nitrogen-helium mixture pressure $p=16+2=18$ Torr, unperturbed gas velocity $U_{\infty}=50 \mathrm{~m} / \mathrm{s}$, and the chamber cross-section area $s=0.052 \mathrm{~m}^{2}$. And we really observe considerable heating of the facility hous- 
ing at plasma outlet from the chamber. Accordingly, we assume that local temperature increase of scale of tens degrees may be caused by the discharge overheating instability process in the case of essential vibration relaxation by admixture. Such sharply non uniform plasma heating would be sufficient to influence the von Karman streets interaction in the critical area of $\lambda \approx 1$. Here, it should be mentioned that a "seed" for the development of this overheating process is available. Really, this seed is local temperature jump at the cathode boundary layer separating. An estimate of this jump with respect to practically unheated surrounding gas is as follows: $\Delta T_{b l} \sim 27 \mathrm{~K}$, whereas the thickness of separating dynamic boundary layer is of value $\delta \sim 0.67 \mathrm{~mm}$ for the cathode diameter $d=4 \mathrm{~mm}$. This estimate is realized according to reference [34] for the case of $100 \%$ efficiency of the cathode water-cooling, i.e. it was assumed that the temperature of the cathode surface was equal to the temperature of unperturbed incoming gas $T_{\text {cath }}=T_{\infty}$. Here the cathode drop was as $208 \mathrm{~V}$ and its thickness as $p d \sim 4 \mathrm{~mm}$. Torr . The whole electric power in cathode fall was assumed to release as heat.

\section{Results and Conclusions}

At present the study of turbulent plasma wake behind a number of cylinders is in progress. Simple, easily treated, and physically justified model of two cylinders wake in the side-by-side arrangement has been proposed. Actual variant of this two-parametric model covers four global wake modes as well as the intermittent regime. This model works well for the case of weak coupling of the streets, and works worse at strong coupling. Nevertheless, within the framework of advanced model, the observed wake intermittent modes were identified for the pitch ratio value $T=L / D \approx 2.2$ studied. And mechanism of glow discharge effect on cylinders wake has been proposed within the framework of the model. It bases on the wake symmetry breaking in plasma overheating process. According to the results obtained and investigations planned the version of two-point transducer for velocity oscillation measurement in both plasma and gas wakes was elaborated. The experimental apparatus in combination with advanced model provide a consistent tool for further plasma wake investigation. Work is under way for control the fluctuations in gas flow incoming the facility chamber. In closing it may be said that now the alternation is of special interest [35], whereas advanced model is an intermittent wake model appropriated for the case of "external" triggered alternation by incoming flow fluctuations.

Main part of these results was briefly presented at the 26-th Symposium on Plasma Physics and Technology, Prague, June 2014.

\section{Acknowledgements}

Discussions with Prof. Eduard V. Teodorovich, numerical calculation by Kirill Yu. Osipenko and electronic circuitry work by Aleksandr A. Sirotin are gratefully acknowledged. This study was supported by The Russian Fund for the Basic Researches, grant 13-01-00742.

\section{References}

[1] Sumner, D. (2010) Journal of Fluids and Structures, 26, 849-899. http://dx.doi.org/10.1016/j.jfluidstructs.2010.07.001

[2] Alam, Md.M., Zhou, Y. and Wang, X.W. (2011) Journal of Fluid Mechanics, 669, 432-471. http://dx.doi.org/10.1017/S0022112010005288

[3] Peng, Y.F. (2013) Journal of Modern Physics, 4, 89-95. http://dx.doi.org/10.4236/jmp.2013.45B015

[4] Bao, Y., Zhou, D. and Tu, J. (2013) Computers \& Fluids, 71, 124-145. http://dx.doi.org/10.1016/j.compfluid.2012.10.013

[5] Verma, A. and Mittal, S. (2011) Physics of Fluids, 23, Article ID: 121701. http://dx.doi.org/10.1063/1.3664869

[6] Zhao, M. and Cheng, L. (2014) Journal of Fluid Mechanics, 751, 1-37. http://dx.doi.org/10.1017/jfm.2014.268

[7] Assi, G.R.S., Bearman, P.W. and Meneghini, J.R. (2010) Journal of Fluid Mechanics, 66, 365-401. http://dx.doi.org/10.1017/S0022112010003095

[8] Song, L. and Wu, S. (2013) Applied Mechanics and Materials, 275-277, 482-485. http://dx.doi.org/10.4028/www.scientificnet/AMM.275-277.482

[9] Luzzatto-Fegiz, P. and Williamson, C.H.K. (2012) Physics of Fluids, 24, Article ID: 066602. http://dx.doi.org/10.1063/1.4724307

[10] Choi, H., Jeon, W-P. and Kim, J. (2008) Annual Review of Fluid Mechanics, 40, 113-139. 
http://dx.doi.org/10.1146/annurev.fluid.39.050905.110149

[11] Corke, T.C., Enloe, C.L. and Wilkinson, S.P. (2010) Annual Review of Fluid Mechanics, 42, 505-529. http://dx.doi.org/10.1146/annurev-fluid-121108-145550

[12] Moreau, E. (2007) Journal of Physics D: Applied Physics, 40, 605-636. http://dx.doi.org/10.1088/0022-3727/40/3/S01

[13] Jukes, T.N. and Choi, K.S. (2009) Physics of Fluids, 21, Article ID: 084103. http://dx.doi.org/10.1063/1.3194307

[14] Sosa, R., Artana, G., Benard, N. and Moreau, E. (2011) Experiments in Fluids, 51, 853-860. http://dx.doi.org/10.1007/s00348-011-1108-0

[15] Sidorenko, A.A., Budovskii, A.D., Postnikov, B.V., Zverkov, I.D., Zanin, B.Yu., Kozlov, V.V. and Maslov, A.A. (2010) Technical Physics Letters, 36, 304-307. http://dx.doi.org/10.1134/S106378501004005X

[16] Raizer, Yu.P. (1991) Gas Discharge Physics. Springer-Verlag, Berlin.

[17] Panchenko, V.Ya., Zavalov, Yu.N., Galushkin, M.G., Grishaev, R.V., Golubev, V.S. and Dubrov, V.D. (2006) Laser Physics, 16, 40-51. http://dx.doi.org/10.1134/S1054660X0601004X

[18] Huang, H. and Wang, Y. (2010) Optical Engineering, 49, Article ID: 114201. http://dx.doi.org/10.1117/1.3509163

[19] Osipov, A.I., Uvarov, A.V. and Vinnichenko, N.A. (2006) Physics of Fluids, 18, Article ID: 105106. http://dx.doi.org/10.1063/1.2364261

[20] Gembarzhevskii, G.V. (2009) Technical Physics Letters, 35, 241-244. http://dx.doi.org/10.1134/S1063785009030146

[21] Siddon, T.E. and Ribner, H.S. (1965) AIAA Journal, 3, 747-749. http://dx.doi.org/10.2514/3.2963

[22] Peschard, I. and Le Gal, P. (1996) Physical Review Letters, 77, 3122-3125. http://dx.doi.org/10.1103/PhysRevLett.77.3122

[23] Landa, P.S. (1996) Nonlinear Oscillations and Waves in Dynamical Systems. Kluwer Academic Publishers, Dordrecht.

[24] Albarede, P. and Provansal, M. (1995) Journal of Fluid Mechanics, 291, 191-222. http://dx.doi.org/10.1017/S0022112095002679

[25] Gembarzhevskii, G.V. (2009) The Effect of Flow Structure Transformation Induced by Electric Discharge and Its Simple Model. Contributed Papers, VI International Conference on Plasma Physics and Plasma Technology, Vol. 1, Minsk, 28 September-2 October 2009, 27-30.

[26] Nayfeh, A.H. (1981) Introduction to Perturbation Techniques. John Wiley \& Sons, Hoboken.

[27] Gembarzhevskii, G.V. and Lednev, A.K. (2013) Reduced Order Model for Effect of Pulsation Energy Redistribution in Cylinders Wake Induced by Glow Discharge. Proceedings of the 12th Workshop on Magneto-Plasma Aerodynamics, Moscow, 26-28 March 2013, 41-44.

[28] Pikovsky, A., Rosenblum, M. and Kurths, J. (2002) Synchronization: A Universal Concept in Nonlinear Science. Cambridge University Press, Cambridge.

[29] Williamson, C.H.K. (1985) Journal of Fluid Mechanics, 159, 1-18. http://dx.doi.org/10.1017/S002211208500307X

[30] Gembarzhevskii, G.V. (2011) Technical Physics Letters, 37, 19-22. http://dx.doi.org/10.1134/S1063785011010020

[31] Kim, H.J. and Durbin, P.A. (1988) Journal of Fluid Mechanics, 196, 431-448. http://dx.doi.org/10.1017/S0022112088002769

[32] Le Gal, P., Chauve, M.P., Lima, R. and Rezende, J. (1990) Physical Review A, 41, 4566-4569.

[33] Provansal, M., Mathis, C. and Boyer, L. (1987) Journal of Fluid Mechanics, 182, 1-22. http://dx.doi.org/10.1017/S0022112087002222

[34] Schlichting, H. (1955) Boundary Layer Theory. Pergamon Press Ltd., London.

[35] Budaev, V.P., Savin, S.P. and Zelenyi, L.M. (2011) Physics-Uspekhi, 54, 875-918. http://dx.doi.org/10.3367/UFNe.0181.201109a.0905 
Scientific Research Publishing (SCIRP) is one of the largest Open Access journal publishers. It is currently publishing more than 200 open access, online, peer-reviewed journals covering a wide range of academic disciplines. SCIRP serves the worldwide academic communities and contributes to the progress and application of science with its publication.

Other selected journals from SCIRP are listed as below. Submit your manuscript to us via either submit@scirp.org or Online Submission Portal.
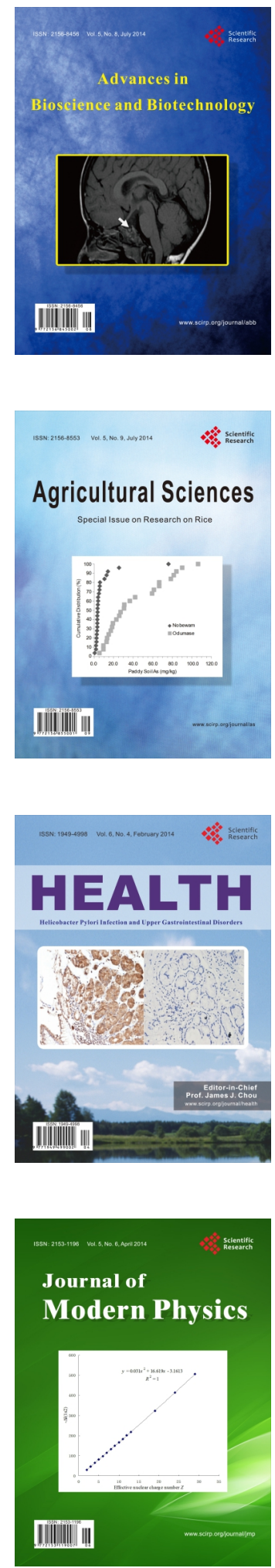
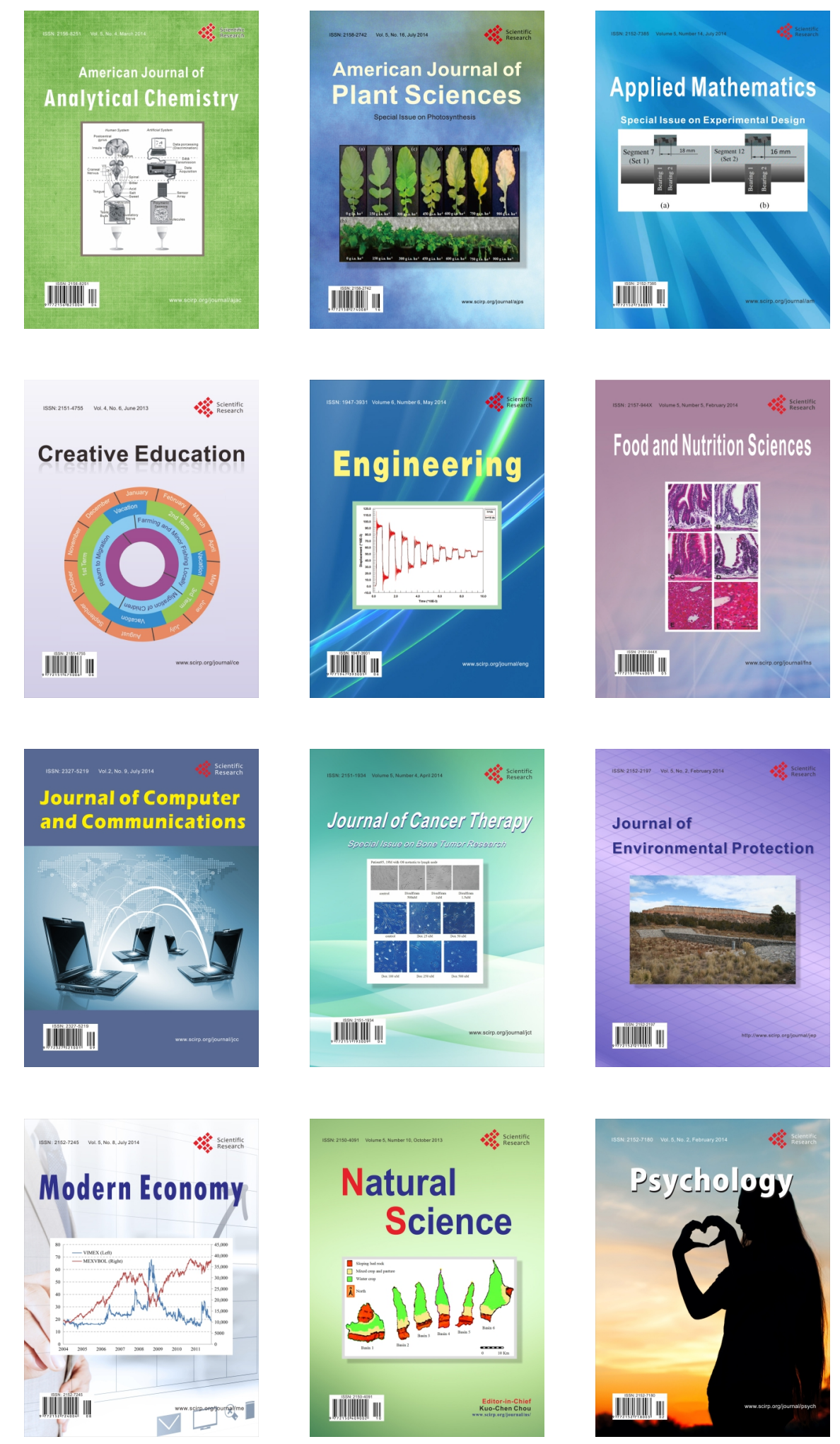